\title{
Seismic behaviour of asymmetric buildings with supplemental damping
}

\author{
Rakesh K. Goel* \\ Department of Civil and Environmental Engineering, Cal Poly State University, San Luis Obispo, CA 93407, U.S.A.
}

\begin{abstract}
SUMMARY
This paper investigated the response of asymmetric-plan buildings with supplemental viscous damping to harmonic ground motion using modal analysis techniques. It is shown that most modal parameters, except dynamic amplification factors (DAFs), are affected very little by the plan-wise distribution of supplemental damping in the practical range of system parameters. Plan-wise distribution of supplemental damping significantly influences the DAFs, which, in turn, influence the modal deformations. These trends are directly related to the apparent modal damping ratios; the first modal damping ratio increases while the second decreases as CSD moves from right to left of the system plan, and their values increase with larger plan-wise spread of the supplemental damping. The largest reduction in the flexible edge deformation occurs when damping in the first mode is maximized by distributing the supplemental damping such that the damping eccentricity takes on the largest value with algebraic sign opposite to the structural eccentricity. Copyright (C) 2000 John Wiley \& Sons, Ltd.
\end{abstract}

KEY WORDS: asymmetric buildings; earthquake response; earthquake behaviour; passive control; plan asymmetry; protective systems; seismic response; supplemental damping; torsion; viscous damping

\section{INTRODUCTION}

Recognizing that asymmetric-plan buildings are especially vulnerable to earthquakes, numerous investigations in the past have focused on the earthquake behaviour of such systems [1-3]. As a result of these studies, procedures to account for undesirable effects of plan asymmetry, such as increased force and ductility demands on lateral load-resisting elements, have been developed and incorporated into many seismic codes [4]. However, control of excessive earthquake-induced deformations in asymmetric-plan buildings has not received much attention. The excessive deformations may lead to premature failure of brittle, non-ductile elements and may result in a sudden loss of the building's strength and stiffness leading to eventual failure. Excessive edge

\footnotetext{
* Correspondence to: Rakesh K. Goel, Department of Civil and Environmental Engineering, California Polytechnic State University, San Luis Obispo, CA 93407, U.S.A.
}

Contract grant sponsor: National Science Foundation; contract grant number: CMS-9812414 
deformation may also cause pounding between closely spaced adjacent buildings, and result in increased second-order $(P-\Delta)$ effects.

Although the effectiveness of supplemental damping in reducing the earthquake response of structures is now well established [5-13], the focus in the past has been on the seismic behaviour of symmetric-plan systems. A few recent investigations have been concerned with the seismic behavior of asymmetric-plan systems with supplemental devices [14-21]. Among these investigations is our previous work reported in a series of papers [18-21]. In this work on the seismic behaviour of linearly elastic, one-storey, asymmetric-plan systems with supplemental viscous damping devices, three additional system parameters were identified: (1) the damping ratio due to supplemental damping devices, $\zeta_{\text {sd }} ;(2)$ the normalized supplemental damping eccentricity, $\bar{e}_{\text {sd }}$; and (3) the normalized supplemental damping radius of gyration, $\bar{\rho}_{\mathrm{sd}}$. Subsequently, the effects of these parameters on the flexible and stiff edges of asymmetric-plan systems subjected to a selected earthquake ground motion were investigated. It was shown that supplemental damping reduces edge deformations, and that the degree of reduction strongly depends on the plan-wise distribution of the supplemental damping. In particular, it was found that asymmetric distribution of the supplemental damping leads to a higher reduction in edge deformations as compared to symmetric distribution. The largest reduction in the flexible edge deformation occurs when $\bar{e}_{\mathrm{sd}}$ takes on the largest negative value, whereas the largest reduction in the stiff edge deformation occurs when $\bar{e}_{\text {sd }}$ takes on the largest positive value. The reduction increases as $\bar{\rho}_{\text {sd }}$ becomes large. It was also shown that edge deformations in asymmetric-plan systems can be reduced to levels equal to or smaller than those of the same edges in the corresponding symmetric-plan system by proper selection of the supplemental damping parameters alone, without redistributing the stiffness and/or mass properties of the system.

While the previous work [18-21] clearly demonstrated the importance of plan-wise distribution of supplemental damping, there is a need to develop a more fundamental understanding of the reasons that lead to reduction in edge deformations. This is important for development of simplified procedures for use in the design practice. With the aim of filling this need, the objectives of this paper are to: (1) develop the necessary theoretical background for modal analysis of asymmetric-plan buildings with supplemental viscous damping, and (2) systematically investigate how various modal parameters and deformations are affected by the plan-wise distribution of supplemental damping.

For this purpose, dynamic response of a linear-elastic, one-storey, one-way symmetric system to harmonic ground motion is investigated using complex-domain modal analysis techniques. Presented first is the theoretical background necessary for modal analysis in the complex domain, followed by description of the system and related parameters. Subsequently, the effects of plan-wise distribution of supplemental damping on the modal parameters are investigated. Finally, the effects on modal deformations at the centre of mass and at the two extreme edges are examined.

\section{THEORETICAL BACKGROUND}

\section{Equations of motion}

Equations of motion for a system subjected to ground motion are:

$$
\mathbf{M u ̈}(t)+\mathbf{C} \dot{\mathbf{u}}(t)+\mathbf{K u}(t)=-\mathbf{M r} \ddot{u}_{\mathrm{g}}(t)
$$


in which matrices $\mathbf{M}, \mathbf{C}$, and $\mathbf{K}$ characterize the mass, damping and stiffness related to the deformations $\mathbf{u}(t)$ at various degrees of freedom; $\mathbf{r}$ is the influence vector; and $\ddot{u}_{\mathrm{g}}(t)$ is the ground acceleration. For a system with $N$ degrees-of-freedom (DOF), $\mathbf{M}, \mathbf{C}$, and $\mathbf{K}$ are $N \times N$ matrices; and $\mathbf{u}(t)$ and $\mathbf{r}$ are $N \times 1$ vectors. Alternatively, the equations of motion can be written in a state-space form as

$$
\mathbf{A} \dot{\mathbf{z}}(t)+\mathbf{B z}(t)=\mathbf{R} \ddot{u}_{\mathrm{g}}(t)
$$

where $z(t)=\langle\mathbf{u}(t) \dot{\mathbf{u}}(t)\rangle^{\mathrm{T}}$ is a $2 N \times 1$ vector; $\mathbf{A}$ and $\mathbf{B}$ are the $2 N \times 2 N$ parameter matrices for the system given by

$$
\mathbf{A}=\left[\begin{array}{cc}
-\mathbf{K} & \mathbf{0} \\
\mathbf{0} & \mathbf{M}
\end{array}\right] \text { and } \mathbf{B}=\left[\begin{array}{ll}
\mathbf{0} & \mathbf{K} \\
\mathbf{K} & \mathbf{C}
\end{array}\right]
$$

and $\mathbf{R}$ is a $2 N \times 1$ vector defined as

$$
\mathbf{R}=\left\{\begin{array}{c}
\mathbf{0} \\
-\mathbf{M r}
\end{array}\right\}
$$

Although the parametric matrices $\mathbf{A}$ and $\mathbf{B}$ in the state-space formulations can be expressed in several other forms [22, 23], the form presented in Equation (3) is appealing because it preserves symmetry of these matrices, and because it does not require, unlike some other forms, inverse of the mass matrix, $\mathbf{M}^{-1}$.

\section{Eigenvalue problem}

The eigenvalue problem for a multi-degree-of-freedom (MDF), undamped system is defined as

$$
\left(\mathbf{K}-\omega^{2} \mathbf{M}\right) \Phi=0
$$

which leads to $N$ values of natural vibration frequencies, $\omega_{\mathrm{n}}$, and vibration modes, $\Phi_{\mathrm{n}}$. Since both $\mathbf{M}$ and $\mathbf{K}$ in structural engineering applications are symmetric and positive definite, both $\omega_{\mathrm{n}}$ and $\Phi_{\mathrm{n}}$ are real valued. For an undamped (or proportionally damped) system, the mode shapes thus obtained may be used to convert the system of $N$ coupled, second-order differential equations (Equation (1)) into a system of $N$ uncoupled, second-order differential equations. The standard modal analysis techniques [22-25] may then be used to solve for time-varying responses of the system to ground motion (or any other type of excitation).

The equations of motion cannot be uncoupled using the undamped mode shapes if the damping matrix is nonproportional, i.e. $\mathbf{C}$ is not proportional to either $\mathbf{M}$ or $\mathbf{K}$ or a combination of both. For systems with non-proportional damping matrix, mode shapes that will uncouple the equations of motion may be obtained from the damped eigenvalue problem

$$
\left(\mathbf{K}+\mathrm{j} \omega \mathbf{C}-\omega^{2} \mathbf{M}\right) \Phi=0
$$

in which $\mathrm{j}=\sqrt{-1}$. Equation (6) represents a quadratic eigenvalue problem and its solution gives $2 N$ eigenvectors and eigenvalues that will, in general, be complex valued. The quadratic 
eigenvalue problem may also be transformed to a linearized form

$$
(\mathbf{B}+\lambda \mathbf{A}) \Phi=0
$$

by utilizing the state-space formulation (Equation (2)). This form also leads to $2 N$ complex-valued eigenvalues $\lambda_{n}$ and eigenvectors $\Phi_{n}$.

The complex eigenvalues $\lambda_{n}$ appear in complex conjugate pairs in the form of

$$
\lambda_{n}=-\zeta_{n} \omega_{n}-\mathrm{j} \omega_{n} \sqrt{1-\zeta_{n}^{2}} \text { and } \lambda_{n}^{*}=-\zeta_{n} \omega_{n}+\mathrm{j} \omega_{n} \sqrt{1-\zeta_{n}^{2}}
$$

in which $\omega_{n}$ and $\zeta_{n}$ are the apparent natural vibration frequency and apparent modal damping ratio, respectively, associated with the $n$th modal pair. Equation (8) may be utilized to obtain the apparent vibration frequencies and apparent modal damping ratios as

$$
\omega_{n}=\sqrt{\operatorname{Re}\left(\lambda_{n}\right)^{2}+\operatorname{Im}\left(\lambda_{n}\right)^{2}} \text { and } \zeta_{n}=\frac{-\operatorname{Re}\left(\lambda_{n}\right)}{\sqrt{\operatorname{Re}\left(\lambda_{n}\right)^{2}+\operatorname{Im}\left(\lambda_{n}\right)^{2}}}
$$

The complex-valued eigenvectors also appear in complex conjugate pairs, $\phi_{n}$ and $\phi_{n}^{*}$. In a complex-valued eigenvector, each element describes the relative magnitude and phase of the motion of the DOF associated with that element when the system is excited at that mode only. In general, the relative position of each DOF can be out of phase by the amount indicated by the complex part of the mode shape element; all DOF vibrate with the same phase angle if the mode shape is real-valued.

\section{Modal analysis of non-proportionally damped systems}

The response vector $\mathbf{z}(t)$ may be expressed as a superposition of the responses in individual modes as

$$
\mathbf{z}(t)=\sum_{i=1}^{2 N} \mathbf{z}_{i}=\sum_{i=1}^{2 N} q_{i} \Phi_{i}
$$

where $q_{i}$ is a complex-valued modal coordinate. Substituting Equation (10) in Equation (2), pre-multiplying by $\Phi_{n}^{\mathrm{T}}$, and utilizing the orthogonality properties of mode shapes gives the following $2 \mathrm{~N}$ uncoupled, first-order differential equations:

$$
\dot{q}_{n}(t)+\lambda_{n} q_{n}(t)=\Gamma_{n} \ddot{u}_{\mathrm{g}}(t), \quad n=1,2, \ldots, 2 N
$$

where

$$
\Gamma_{n}=\frac{\Phi_{n}^{\mathrm{T}} \mathbf{R}}{\Phi_{n}^{\mathrm{T}} \mathbf{A} \Phi_{n}}, \quad n=1,2, \ldots, 2 N
$$


is the modal participation factor. The solution of the first-order differential equation is given by [26]

$$
q_{n}(t)=\Gamma_{n} \mathrm{e}^{-\lambda_{n} t}\left[\int \mathrm{e}^{\lambda_{n} t} \ddot{u}_{\mathrm{g}}(t) \mathrm{d} t+I_{n}\right]
$$

where $I_{n}$ is a constant that depends on the initial conditions. Once the modal coordinates have been determined from Equation (13), the response vector can be computed from Equation (10).

\section{Steady-state response to harmonic ground motion}

Let $\mathbf{z}(t)$ be the steady-state response of the system due to harmonic ground acceleration defined by

$$
\ddot{u}_{\mathrm{g}}(t)=\ddot{u}_{\mathrm{go}} \mathrm{e}^{\mathrm{j} \omega t}
$$

in which $\ddot{u}_{\mathrm{go}}$ is the peak value of the ground acceleration and $\omega$ is the forcing frequency. Since $\mathrm{e}^{\mathrm{j} \omega t}=\cos (\omega t)+\mathrm{j} \sin (\omega t), \operatorname{Re}[\mathbf{z}(t)]$ will be the response to the cosine loading whereas $\operatorname{Im}[\mathbf{z}(t)]$ will be the response to the sine loading. The response $\mathbf{z}(t)$ to the harmonic ground motion of Equation (14) can be computed from the modal analysis method outlined in the preceding section. Following are some of the intermediate steps of this method.

The steady-state modal response coordinates is given as

$$
q_{n}(t)=\Gamma_{n} H_{n} \ddot{u}_{\mathrm{go}} \mathrm{e}^{\mathrm{j} \omega t}
$$

in which the complex frequency response function is

$$
H_{n}=\frac{1}{\lambda_{n}+\mathrm{j} \omega}
$$

The $k$ th element of the response vector due to $n$th mode is then given by

$$
z_{k n}(t)=\Gamma_{n} H_{n} \Phi_{k n} \ddot{u}_{\mathrm{go}} \mathrm{e}^{\mathrm{j} \omega t}
$$

and the total response is obtained by summing the contributions due to all modes (Equation (10)).

Since the modes occur in complex-conjugate pairs, it is convenient to express the response at $k$ th DOF as a summation of responses due to $N$ modal pairs (details are available in the appendix) as

$$
z_{k}(t)=\sum_{n=1}^{N} \hat{z}_{k n}(t)
$$

with $\hat{z}_{k n}(t)$ defined as

$$
\hat{z}_{k n}(t)=\left|\Gamma_{n}\right| \times\left|\Phi_{k n}\right| \times \frac{2 R_{\mathrm{dn}}}{\omega_{n}} \times C_{k n} \times \ddot{u}_{\mathrm{go}} \mathrm{e}^{\mathrm{j}\left(\omega t+\theta_{n}^{R}+\theta_{k n}^{C}\right)}
$$



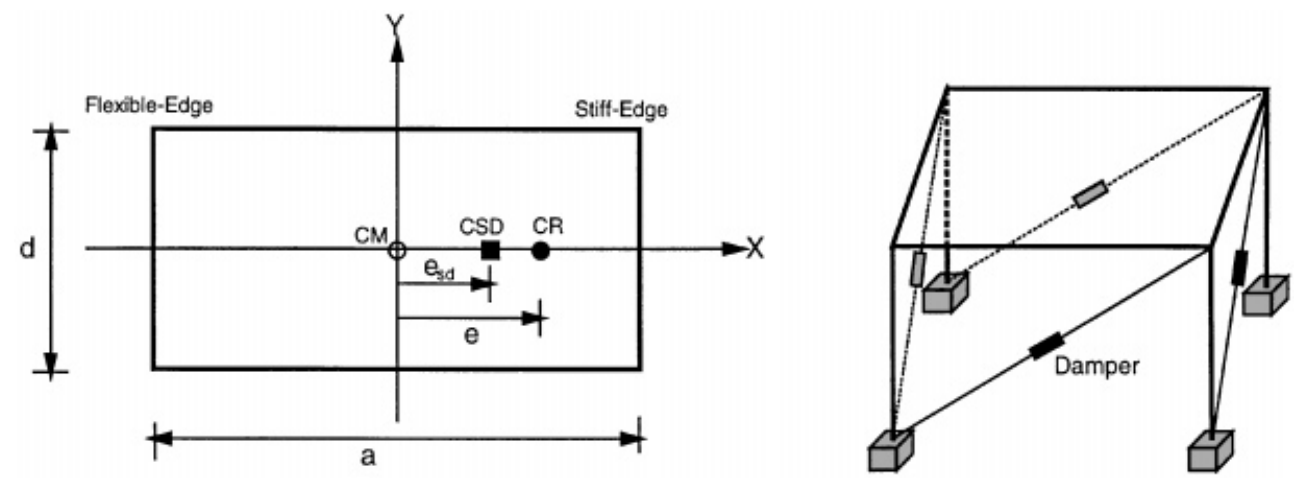

Figure 1. One-storey asymmetric-plan system with supplemental viscous damping.

in which $\left|\Gamma_{n}\right|$ and $\left|\Phi_{k n}\right|$ are magnitudes (or absolute values) of the modal participation factor and the mode shape component, respectively; $R_{\mathrm{dn}}$ is the dynamic amplification factor defined by Equation (A7); $C_{k n}$ is the angular constant defined by Equation (A12); $\theta_{n}^{R}$ is the phase angle defined by Equation (A7); and $\theta_{k n}^{C}$ is the angle defined by Equation (A13).

\section{ASYMMETRIC-PLAN SYSTEM}

\section{One-storey system}

The system considered was the idealized one-storey building of Figure 1 consisting of a rigid deck supported by structural elements (wall, columns, moment-frames, braced-frames, etc.) in each of the two orthogonal directions, and included fluid viscous dampers incorporated into the bracing system. The mass properties of the system were assumed to be symmetric about both the $X$ - and $Y$-axis whereas the stiffness and the damper properties were considered to be symmetric only about the $X$-axis. The distance between the centre of mass (CM) and the centre of supplemental damping (CSD) is denoted by the supplemental damping eccentricity, $e_{\mathrm{sd}}$, whereas distance between the $\mathrm{CM}$ and the centre of rigidity $(\mathrm{CR})$ is defined by the stiffness eccentricities, $e$.

\section{System matrices and parameters}

The one-way symmetric system (Figure 1) has two DOF when subjected to ground motion along the $Y$-axis: translation along the $Y$-axis and rotation about a vertical axis. The displacement vector $\mathbf{u}$ for the system is defined by $\mathbf{u}^{\mathrm{T}}=\left\langle u_{y} \quad a u_{\theta}\right\rangle$ where $u_{y}$ is the horizontal displacement relative to the ground of the $\mathrm{CM}$ along the $Y$-axis, $u_{\theta}$ is the rotation of the deck about a vertical axis, and $a$ is the plan dimension of the system along the $X$-axis. The mass, stiffness, and damping matrices of the system with respect to the DOF $\mathbf{u}$ are then given in terms of the system parameters as

$$
\mathbf{M}=\left[\begin{array}{cc}
m & 0 \\
0 & \frac{1+\alpha^{2}}{12 \alpha^{2}} m
\end{array}\right]
$$


where $m$ is the total deck mass and $\alpha=a / d$ is the aspect ratio of the deck

$$
\mathbf{K}=m \omega_{y}^{2}\left[\begin{array}{cc}
1 & \bar{e} \\
\bar{e} & \bar{e}^{2}+\frac{1+\alpha^{2}}{12 \alpha^{2}} \Omega_{\theta}^{2}
\end{array}\right]
$$

in which $\omega_{y}$ is the undamped transverse vibration frequency of a corresponding uncoupled system defined as a system with coincidental CM and CR but with relative location and stiffness of all resisting elements identical to those in the asymmetric-plan system; $\Omega_{\theta}$ is ratio of the torsional and transverse frequencies of the corresponding uncoupled system; and $\bar{e}=e \div a$,

$$
\mathbf{C}=\mathbf{C}_{n}+\mathbf{C}_{\mathrm{sd}}
$$

where $\mathbf{C}_{n}$ is a proportional damping matrix defined as

$$
\mathbf{C}_{n}=a_{0} \mathbf{M}+a_{1} \mathbf{K}
$$

in which constants $a_{0}$ and $a_{1}$ depend on damping ratios in the two undamped vibration modes of the system, and $\mathbf{C}_{\mathrm{sd}}$ is the damping matrix due to supplemental dampers given as

$$
\mathbf{C}_{\mathrm{sf}}=2 m \omega_{y} \zeta_{\mathrm{sd}}\left[\begin{array}{cc}
1 & \bar{e}_{\mathrm{sd}} \\
\bar{e}_{\mathrm{sd}} & \bar{e}_{\mathrm{sd}}^{2}+\bar{\rho}_{\mathrm{sd}}^{2}
\end{array}\right]
$$

in which $\zeta_{\text {sd }}$ is the supplemental damping ratio; $\bar{e}_{\mathrm{sd}}=e_{\mathrm{sd}} \div a ; \bar{\rho}_{\mathrm{sd}}=\rho_{\mathrm{sd}} \div a$. Detailed descriptions of various system parameters and derivations of the system matrices are available elsewhere [18].

\section{Selected system parameters}

The following system parameters were considered in this investigation: $\Omega_{\theta}=1$ to represents systems with strong coupling between lateral and torsional motions in the elastic range for which effects of supplemental damping were found to be significant [18]; $\bar{e}=0.2$ which implies an eccentricity of 20 per cent of the plan dimension; $\alpha=2 ; a_{0}$ and $a_{1}$ in Equation (23) to achieve damping ratios in both vibration modes of the system without supplemental damping equal to 5 per cent; and $\zeta_{\text {sd }}=10$ per cent. The $\bar{e}_{\text {sd }}$ was varied between the extreme values of -0.5 to 0.5 . The selected values of $\bar{\rho}_{\text {sd }}=0,0.2$, and 0.5 represent low, medium, and large spreads of the supplemental damping about the CSD.

\section{Vibration properties and responses}

The eigenvalue problem of Equation (7) leads to four complex-valued eigenvalues and eigenvectors for the two DOF asymmetric-plan system of Figure 1. These eigenvalues can then be used to calculate apparent vibration frequencies, $\omega_{1}$ and $\omega_{2}$ (or vibration periods, $T_{1}$ and $T_{2}$ ) and apparent damping ratios, $\zeta_{1}$ and $\zeta_{2}$, by using Equation (9). The complex-valued eigenvectors are given by

$$
\phi_{n}=\left\{\begin{array}{c}
\alpha_{n}^{y} \mathrm{e}^{\mathrm{j} \theta_{n}^{y}} \\
\alpha_{n}^{\theta} \mathrm{e}^{\mathrm{j} \theta_{n}^{\theta}}
\end{array}\right\} \quad \text { and } \quad \phi_{n}^{*}=\left\{\begin{array}{c}
\alpha_{n}^{y} \mathrm{e}^{-\mathrm{j} \theta_{n}^{y}} \\
\alpha_{n}^{\theta} \mathrm{e}^{-\mathrm{j} \theta_{n}^{\theta}}
\end{array}\right\}
$$




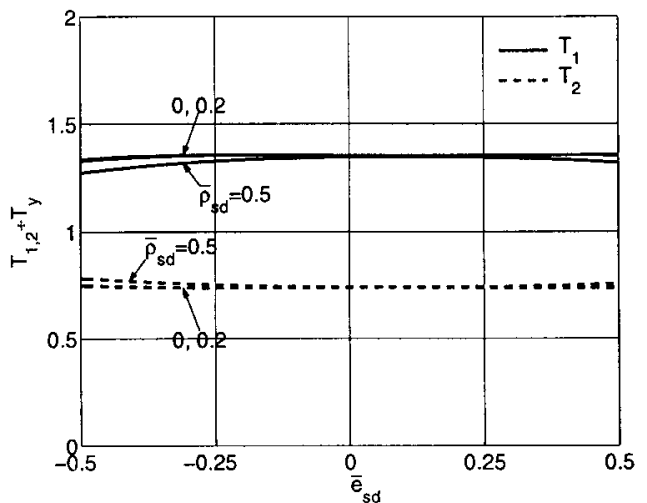

(a)

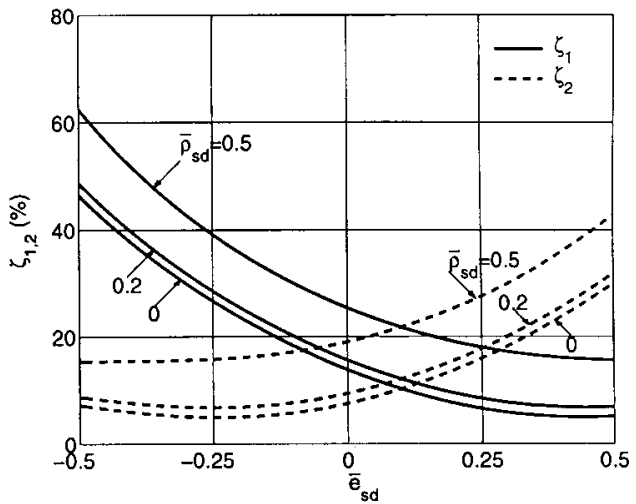

(b)

Figure 2. Apparent vibration properties of asymmetric-plan systems with supplemental damping: (a) periods; and (b) damping ratios.

where $\alpha_{n}^{y}$ and $\alpha_{n}^{\theta}$ are the translational and rotational components, respectively, and $\theta_{n}^{y}$ and $\theta_{n}^{\theta}$ the associated phase angles. For systems with non-proportional damping the phase angles between two elements of the same eigenvector will be different, i.e. $\theta_{n}^{y} \neq \theta_{n}^{\theta}$. For systems without damping (or proportional damping), on the other hand, the phase angles would be the same, i.e. $\theta_{n}^{y}=\theta_{n}^{\theta}$; in this case, the eigenvector can also be normalized such that $\theta_{n}^{y}=\theta_{n}^{\theta}=0$, i.e. the eigenvectors are real-valued. The eigenvectors in this investigation were normalized such that $\Phi_{n}^{\mathrm{T}} \mathbf{A} \Phi_{n}=1$.

\section{EFFECTS OF SYSTEM PARAMETERS ON MODAL PROPERTIES}

Prior to investigating the effects of the plan-wise distribution of supplemental damping on the dynamic response of asymmetric-plan systems, it is useful to examine how supplemental damping affects the various modal properties: apparent modal periods, apparent damping ratios, mode shape components, modal participation factors, and dynamic amplification factors. Therefore, variations of the modal properties for values of $\bar{e}_{\mathrm{sd}}$ in the range of -0.5 to 0.5 and three values of $\bar{\rho}_{\text {sd }}=0,0.2$, and 0.5 were computed and presented in Figures $2-8$. These results permit the following observations.

\section{Periods}

The apparent modal periods are affected very little by plan-wise distribution of supplemental damping (Figure 2(a)) which becomes apparent from little variation in the normalized values of the two periods with $\bar{e}_{\mathrm{sd}}$ and almost identical curves for the three values of $\bar{\rho}_{\mathrm{sd}}$. This trend is consistent with the expectation that vibration period would be practically independent of the system damping. 


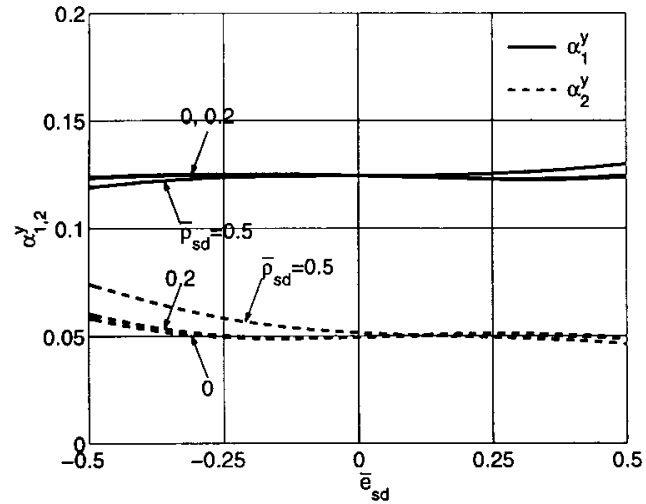

(a)

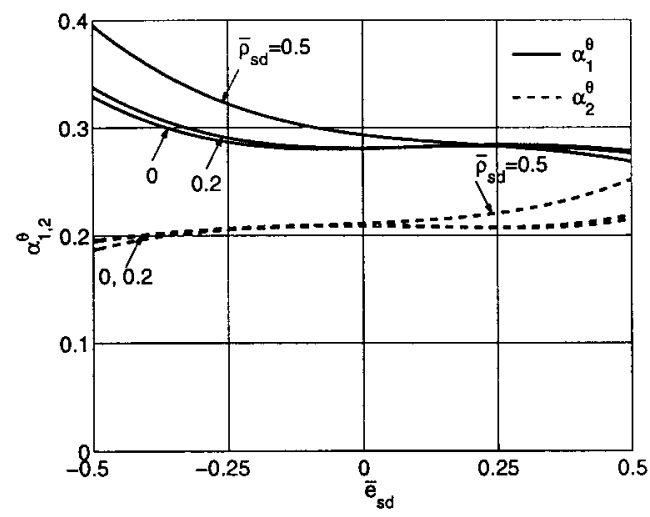

(c)

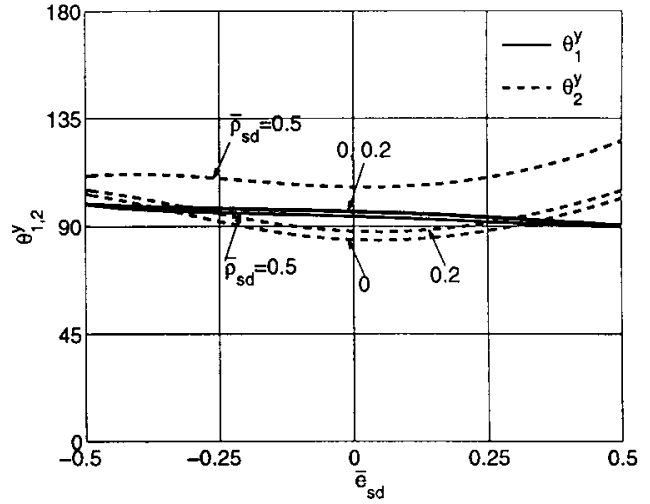

(b)

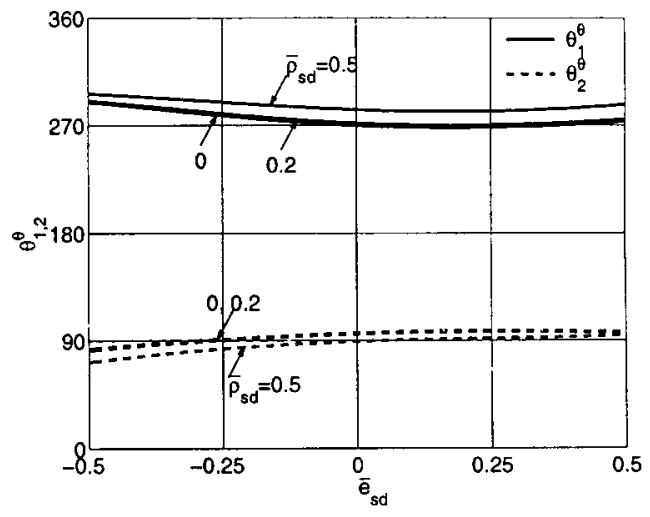

(d)

Figure 3. Mode shapes of asymmetric-plan systems with supplemental damping: (a) magnitude for translational component; (b) phase angle for translational component; (c) magnitude for rotational component; and (d) phase angle for rotational component.

\section{Damping ratios}

The apparent modal damping ratios are significantly affected by both $\bar{e}_{\text {sd }}$ and $\bar{\rho}_{\text {sd }}$ (Figure 2(b)). In particular, $\zeta_{1}$ decreases and $\zeta_{2}$ increases as the CSD moves from left to right in the system plan, i.e. $\bar{e}_{\text {sd }}$ varies from -0.5 to 0.5 , and both $\zeta_{1}$ and $\zeta_{2}$ become larger as $\bar{\rho}_{\text {sd }}$ increases.

The presented results show that damping ratios much higher than the damping obtained by evenly distributing the supplemental damping in the system plan, i.e. $\bar{e}_{\mathrm{sd}}=0$, are possible. Consider, for example, the damping ratios in systems with $\bar{\rho}_{\text {sd }}=0.5$. The apparent value of $\zeta_{1}$ is nearly two-and-a-half times for $\bar{e}_{\text {sd }}=-0.5$ compared that for $\bar{e}_{\text {sd }}=0$; the two values are 62 and 


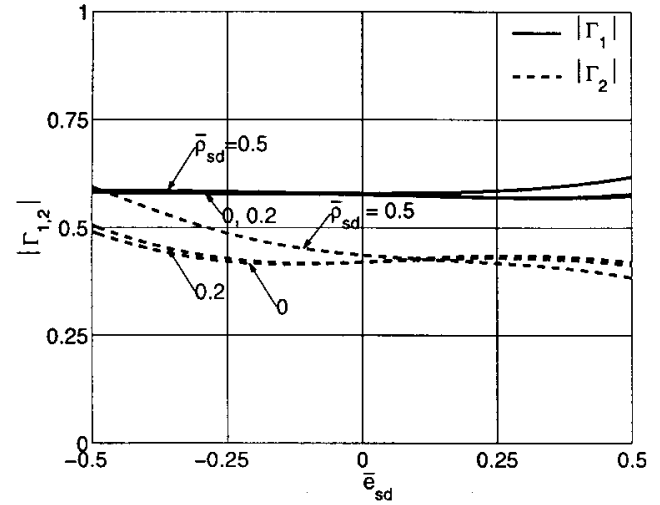

(a)

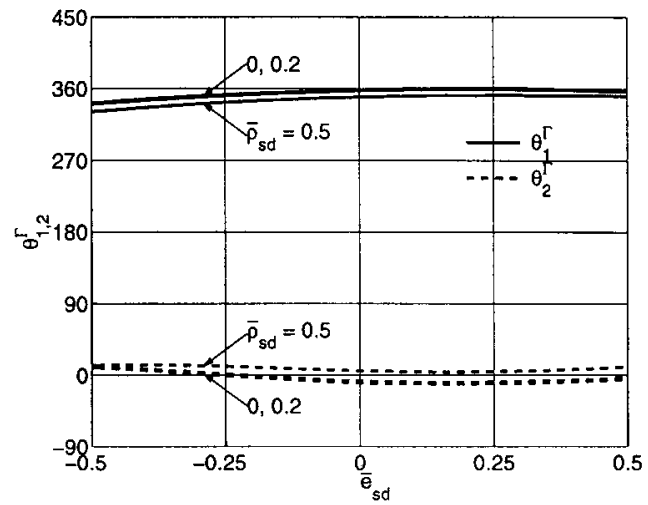

(b)

Figure 4. Modal participation factors for asymmetric-plan systems with supplemental damping: (a) magnitude; and (b) phase angle.

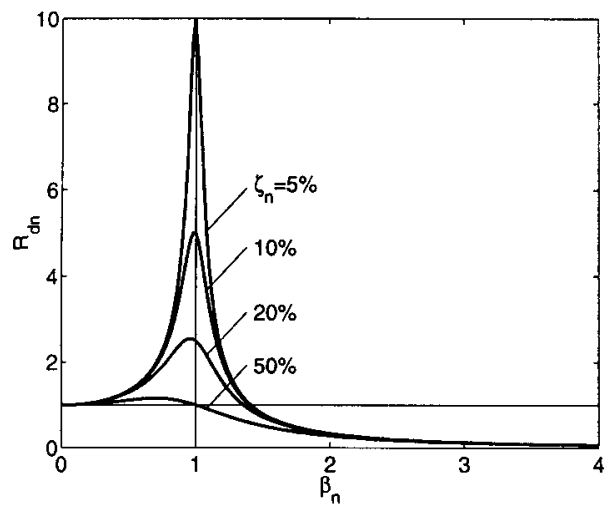

(a)

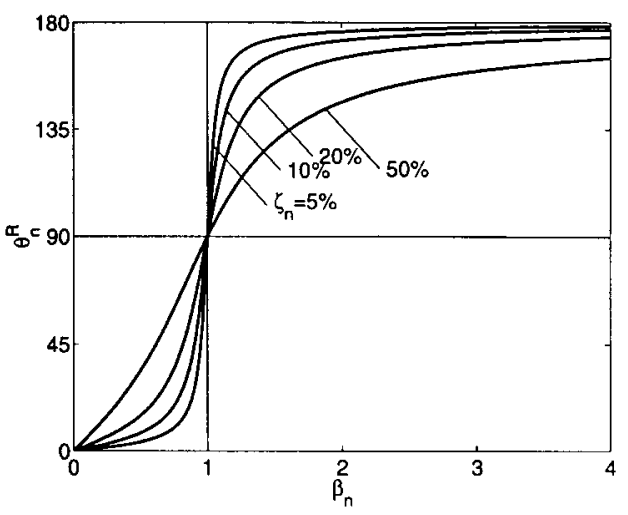

(b)

Figure 5. Dynamic response (or amplification) factor: (a) magnitude; and (b) phase angle.

25 per cent, respectively. Similarly, the apparent value of $\zeta_{2}$ is more than two times for $\bar{e}_{\mathrm{sd}}=0.5$ compared to that for $\bar{e}_{\mathrm{sd}}=0$; the two values are 43 and 19 per cent, respectively.

It is also apparent that damping ratios much higher than those in the corresponding symmetricplan system are possible with appropriate plan-wise distribution of the supplemental damping. For example, a total of 15 per cent damping ( 5 per cent natural +10 per cent supplemental) in the symmetric system may give up to 62 per cent in the fundamental modal pair of asymmetric-plan system with careful plan-wise distribution of the supplemental damping. 


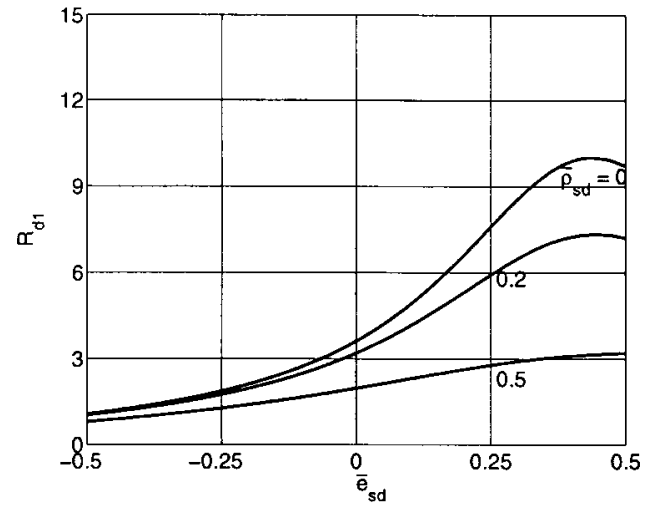

(a)

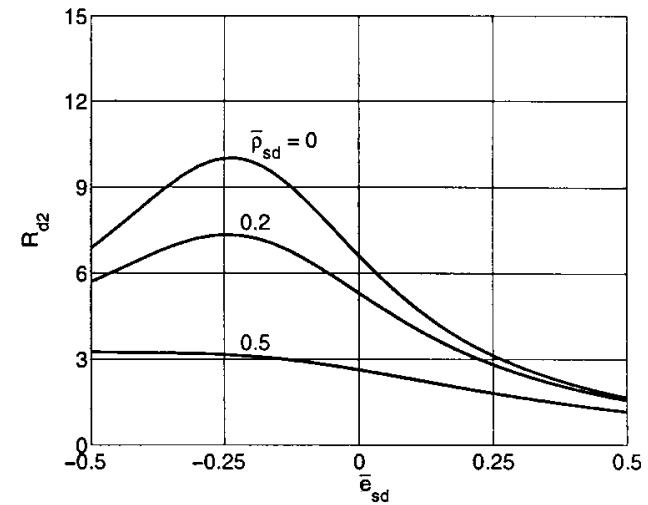

(b)

Figure 6. Dynamic response (or amplification) factor for asymmetric-plan systems with supplemental damping: (a) $\beta_{1}=1$ and (b) $\beta_{2}=1$.

The largest possible values of $\zeta_{1}$ and $\zeta_{2}$ do not occur for the same values of $\bar{e}_{\mathrm{sd}}: \zeta_{2}$ is nearly at its minimum value when $\zeta_{1}$ reaches its maximum value and vice versa. This indicates that the plan-wise distribution of the supplemental damping, i.e., selection of $\bar{e}_{\mathrm{sd}}$, should depend on which of the two modal pairs dominates the response. If the first modal pair dominates, the supplemental damping should be distributed to maximize $\zeta_{1}$ by locating the CSD as far away from the $\mathrm{CM}$, on the side opposite to the $\mathrm{CR}$, as possible, i.e. $\bar{e}_{\text {sd }}$ as close to -0.5 as possible. If the second modal pair dominates, then the supplemental damping should be distributed to maximize $\zeta_{2}$ by locating the CSD as far away from the $\mathrm{CM}$, on the same side of the $\mathrm{CR}$, as possible, i.e., $\bar{e}_{\mathrm{sd}}$ as close to 0.5 as possible.

\section{Mode shape elements}

The magnitudes (or absolute value) and phase angles of translational and rotational components of the two complex-valued eigenvector pairs are plotted in Figure 3. These results shows that translational components of the first and second modal pair, $\alpha_{1}^{y}$ and $\alpha_{2}^{y}$, are affected very little either by $\bar{e}_{\text {sd }}$ or by $\bar{\rho}_{\text {sd }}$ (Figure 3(a)), except for systems with $\bar{\rho}_{\text {sd }}=0.5$ for which $\alpha_{2}^{y}$ tends to increase slightly with decreasing values of $\bar{e}_{\mathrm{sd}}$ in the range of $\bar{e}_{\mathrm{sd}}<0$. The trends observed for the magnitude of the mode shape components also apply to the phase angles, $\theta_{1}^{y}$ and $\theta_{2}^{y}$, with only minor differences (Figure 3(b)): $\theta_{2}^{y}$ tends to increase slightly as $\bar{\rho}_{\text {sd }}$ becomes larger over the entire range of $\bar{e}_{\text {sd }}$, as opposed to $\alpha_{2}^{y}$ which becomes larger only for $\bar{e}_{\text {sd }}<0$.

The rotational components of the mode shapes, $\alpha_{1}^{\theta}$ and $\alpha_{2}^{\theta}$, are affected to a much higher degree compared to the translational components by both $\bar{e}_{\mathrm{sd}}$ or by $\bar{\rho}_{\text {sd }}$ (Figure 3(c)). In the range of $\bar{e}_{\mathrm{sd}}<0, \alpha_{2}^{\theta}$ reduces with decreasing values of $\bar{e}_{\mathrm{sd}}$ and $\bar{\rho}_{\mathrm{sd}}$. Furthermore, curves for different values of $\bar{\rho}_{\text {sd }}$ may cross over in the range of $\bar{e}_{\text {sd }}>0$. The trends for $\alpha_{2}^{\theta}$ are generally opposite to those for $\alpha_{1}^{\theta}$ but the variations are much smaller. The phase angles, $\theta_{1}^{\theta}$ and $\theta_{2}^{\theta}$, are affected only slightly by $\bar{e}_{\text {sd }}$ or by $\bar{\rho}_{\text {sd }}($ Figure $3(\mathrm{~d}))$. In the range of $\bar{e}_{\mathrm{sd}}<0, \theta_{1}^{\theta}$ tends to decrease and $\theta_{2}^{\theta}$ tends to increase 


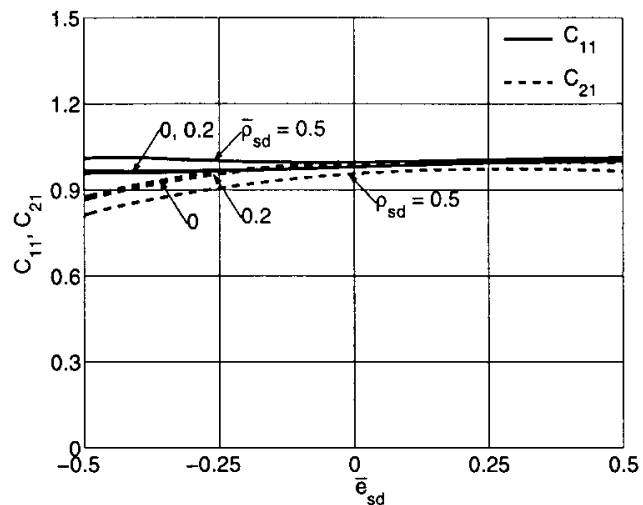

(a)

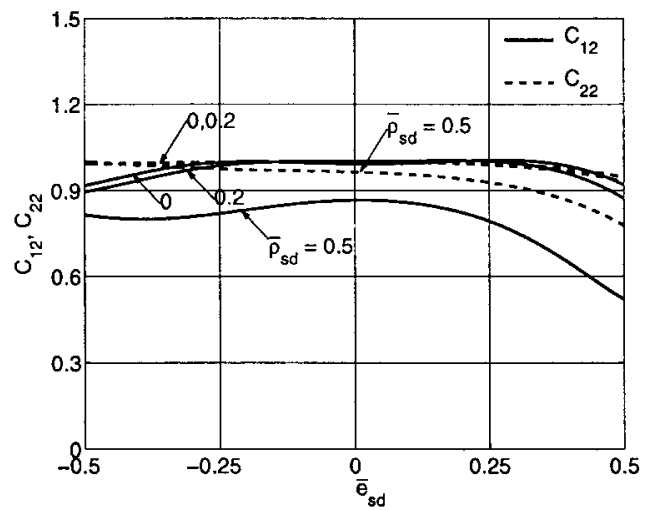

(c)

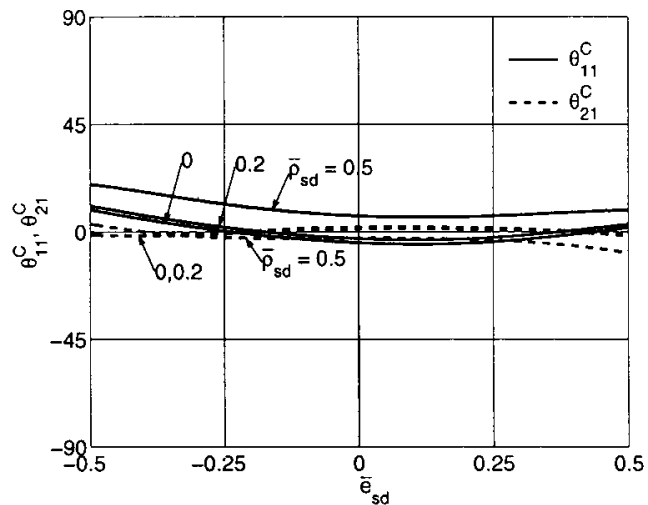

(b)

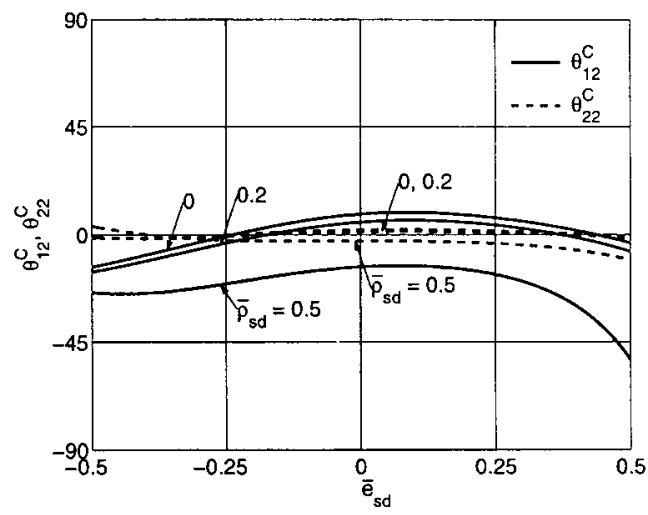

(d)

Figure 7. Angular constant for asymmetric-plan systems with supplemental damping with $\beta_{1}=1$ : (a) magnitude for first mode; (b) phase angle for first mode; (c) magnitude for second mode and (d) phase angle for second mode.

slightly with decreasing values of $\bar{e}_{\text {sd }}$. The curves tend to flatten in the range of $\bar{e}_{\text {sd }}>0$, indicating little dependence of phase angles on $\bar{e}_{\mathrm{sd}}$.

The phase angles of translational and rotational components of the same mode shape of systems with supplemental damping may not be the same, as opposed to systems with no damping (or proportional damping) where the phase angles are the same. This becomes apparent by comparing the results presented in Figure 3(b) and 3(d). For example, for a system with $\bar{e}_{\mathrm{sd}}=-0.5$ and $\bar{\rho}_{\mathrm{sd}}=0.5, \theta_{1}^{y}$ and $\theta_{1}^{\theta}$ differ by about $18^{\circ}: \theta_{1}^{y}$ is about $99^{\circ}$ and $\theta_{1}^{\theta}$ is nearly $117^{\circ}$; if the system had no damping, the two phase angles would be the same. Note that $\theta_{1}^{\theta}=117^{\circ}$ is obtained by subtracting $180^{\circ}$ from the plotted value of $297^{\circ}$ to account for negative algebraic sign of $\alpha_{1}^{\theta}$. 


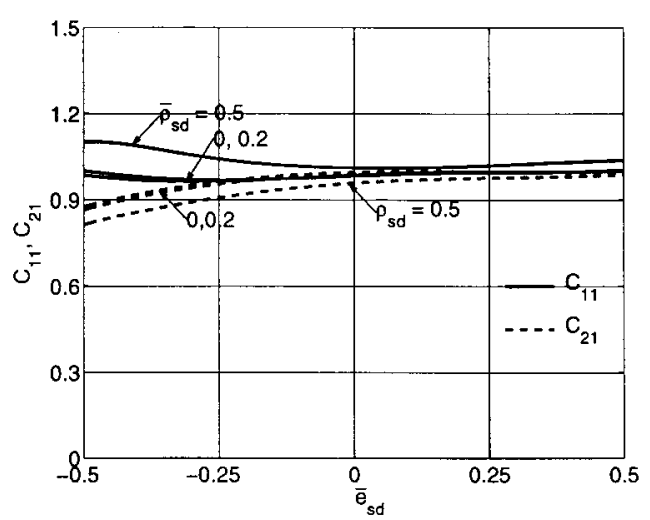

(a)

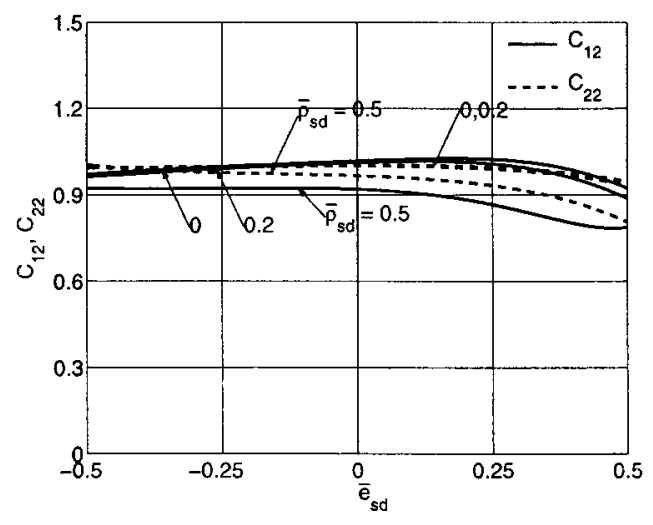

(c)

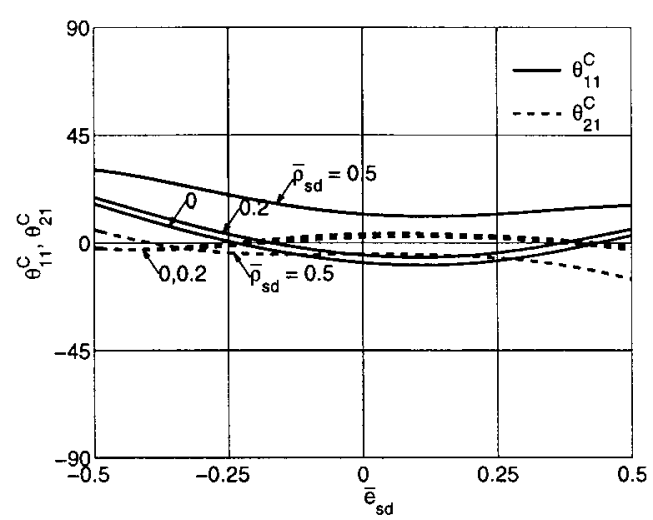

(b)

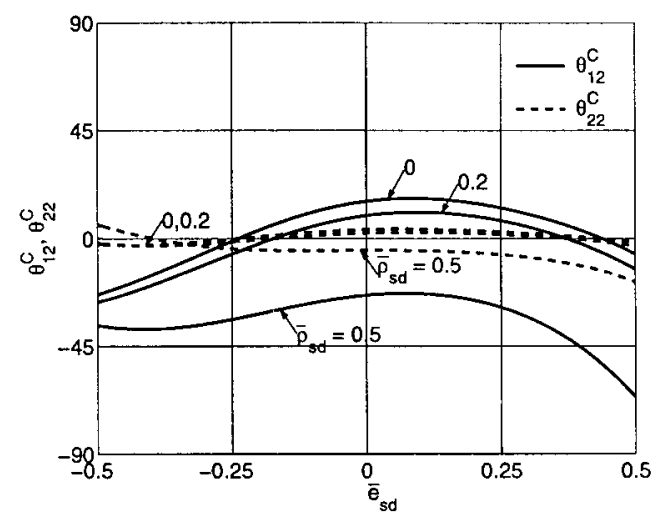

(d)

Figure 8. Angular constant for asymmetric-plan systems with supplemental damping with $\beta_{2}=1$ : (a) magnitude for first mode; (b) phase angle for first mode; (c) magnitude for second mode and (d) phase angle for second mode.

The difference in phase angles of the translational and torsional component depend to a smaller extent on $\bar{e}_{\text {sd }}$ and to a slightly larger degree on $\bar{\rho}_{\text {sd }}$. Although magnitudes of the translational and rotational motions at the $\mathrm{CM}$ are not affected by this difference in phase angles, peak value of the motion at any other location, for example, edges of the deck, may be affected by such differences.

\section{Modal participation factor}

The magnitudes (or absolute value), $\left|\Gamma_{1}\right|$ and $\left|\Gamma_{2}\right|$, and phase angles, $\theta_{1}^{\Gamma}$ and $\theta_{2}^{\Gamma}$, of the two modal participation factors (Equation (12)) associated with the two complex-valued 
mode shapes are plotted in Figure 4. The presented results permit the following observations.

The magnitude of modal participation factor in the first modal pair, $\left|\Gamma_{1}\right|$, is affected very little by plan-wise distribution of the supplemental damping, except for $0.1<\bar{e}_{\text {sd }}<0.5$ and $\bar{\rho}_{\text {sd }}=0.5$ for which $\left|\Gamma_{1}\right|$ tends to increase slightly with increasing $\bar{e}_{\text {sd }}$ (Figure 4(a)). The magnitude of modal participation factor in the second modal pair, $\left|\Gamma_{2}\right|$, may however be affected much more. In particular, $\left|\Gamma_{2}\right|$ decreases as $\bar{e}_{\text {sd }}$ varies from -0.5 to 0 , may increase slightly as $\bar{e}_{\text {sd }}$ takes on larger positive values, and eventually decreases slightly for much larger values of $\bar{e}_{\text {sd }}$. These effects are most prominent for large values of $\bar{\rho}_{\text {sd }}$, i.e., $\bar{\rho}_{\text {sd }}=0.5$.

The phase angles, $\theta_{1}^{\Gamma}$ and $\theta_{2}^{\Gamma}$, are affected slightly by the plan-wise distribution of the supplemental damping. In particular, $\theta_{1}^{\Gamma}$ increases and $\theta_{2}^{\Gamma}$ decreases slightly as $\bar{e}_{\mathrm{sd}}$ varies from -0.5 to 0 and this trend may reverse as $\bar{e}_{\text {sd }}$ varies from 0 to 0.5 . As observed earlier, this effect tends to more prominent for large $\bar{\rho}_{\text {sd }}$.

\section{Dynamic amplification factor}

Figure 5 shows the dynamic amplification factor (DAF), $R_{\mathrm{dn}}$, and the associated phase angle, $\theta_{n}^{R}$, (Equation (A8)) as a function of the frequency ratio, $\beta_{n}$, for different values of damping ratios. DAF represents amplification (or de-amplification) of the steady-state response of a singledegree-of freedom system to harmonic loading and phase angle indicates phase lag between the

loading and the response. Detailed derivation and interpretation of $R_{\mathrm{dn}}$ and $\theta_{1}^{R}$ (Equation (A8)) can be found in any standard textbook on structural dynamics, e.g., [24].

Figure 6 shows the variation of $R_{\mathrm{dn}}$ with the plan-wise distribution of supplemental damping. Results are presented for two cases: $\beta_{1}=1$ (Figure 6(a)) and $\beta_{2}=1$ (Figure 6(b)). In the first case, only $R_{\mathrm{d} 1}$ is presented because $R_{\mathrm{d} 2}$ is much smaller than $R_{\mathrm{d} 1}$; note that $\beta_{2}=\beta_{1} \times T_{2} \div T_{1}<1$ for which $R_{\mathrm{d} 2}$ is nearly equal to one (Figure 5(a)). For similar reasons, only $R_{\mathrm{d} 2}$ is presented in the second case. The results permit the following observations.

For $\beta_{1}=1$ (Figure 6(a)), $R_{\mathrm{d} 1}$ increases as CSD moves from left to right of the system plan, i.e., as $\bar{e}_{\text {sd }}$ varies from -0.5 to 0.5 . The degree to which $R_{\mathrm{d} 1}$ increases depends on $\bar{\rho}_{\mathrm{sd}}$ : larger the value of $\bar{\rho}_{\text {sd }}$, smaller the increase. These trends are nearly opposite to the previous observations on $\zeta_{1}$ which decreases as $\bar{e}_{\text {sd }}$ varies from -0.5 to 0.5 , and becomes larger as $\bar{\rho}_{\text {sd }}$ increases (Figure 2(b)). This is to be expected because $R_{\mathrm{dn}}$ is reduced significantly as damping is increased and vice versa (Figure 5(a)). For $\beta_{2}=1$ (Figure 6(b)), $R_{\mathrm{d} 2}$ decreases as CSD moves from left to right of the system plan, i.e., as $\bar{e}_{\text {sd }}$ varies from -0.5 to 0.5 and $R_{\mathrm{d} 2}$ becomes smaller as value of $\bar{\rho}_{\text {sd }}$ increases. The trends for $R_{\mathrm{d} 2}$ are related to $\zeta_{2}$ which increases as $\bar{e}_{\mathrm{sd}}$ varies from -0.5 to 0.5 , and becomes larger as $\bar{\rho}_{\text {sd }}$ increases (Figure 2(b)).

\section{Angular constant}

Figures 7 and 8 show the variation of the angular constant, $C_{k n}$ (Equation (A13)), and its associated phase angle, $\theta_{k n}^{C}$ (Equation (A14)). The results are presented in Figure 7 for $\beta_{1}=1$ and in Figure 8 for $\beta_{2}=1$. It is apparent from these figures that the angular constant varies very little with $\bar{e}_{\mathrm{sd}}$ except for very large value of $\bar{\rho}_{\mathrm{sd}}$. For large value of $\bar{\rho}_{\mathrm{sd}}(=0.5)$, the angular constant tends to become smaller as $\bar{e}_{\text {sd }}$ varies from 0.5 to -0.5 . This effect is more pronounced for the second mode $\left(C_{12}\right.$ and $\left.C_{22}\right)$ compared to the first mode $\left(C_{11}\right.$ and $\left.C_{21}\right)$. Similar trends also apply to the associated phase angles. 


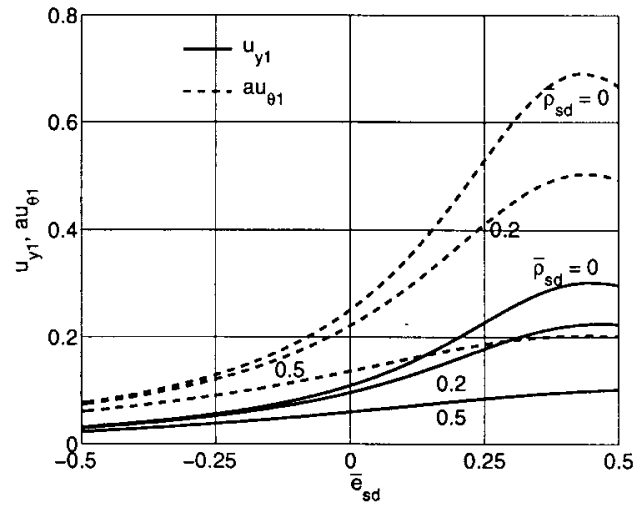

(a)

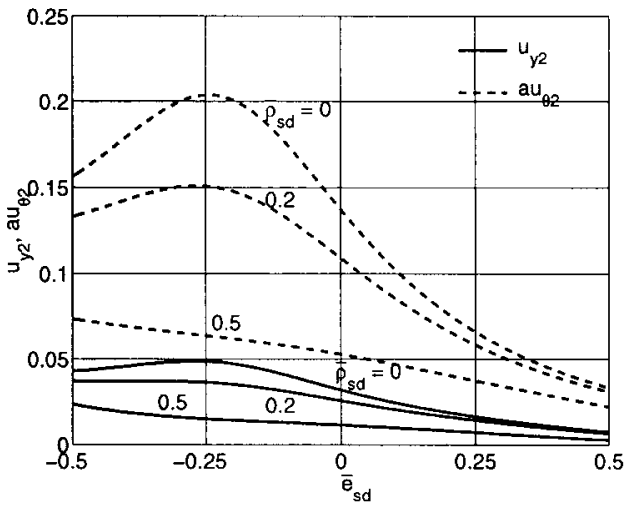

(b)

Figure 9. Deformations at the CM of asymmetric-plan systems with supplemental damping: (a) $\beta_{1}=1$ and (b) $\beta_{2}=1$.

\section{Summary of effects on modal parameters}

The preceding results indicate that among all modal parameters $-\left|\Gamma_{n}\right|,\left|\phi_{k n}\right|, R_{\mathrm{dn}}, \omega_{n}, C_{k n}, \theta_{n}^{R}$, and $\theta_{k n}^{C}$ - that are required to compute the modal response (Equation (19)), $R_{\mathrm{dn}}$ is the one most affected by the plan-wise distribution of supplemental damping; other parameters are influenced to a much smaller degree. This is especially true for the practical values of $\bar{\rho}_{\text {sd }} \leqslant 0.25$. Although, apparent modal damping ratios, $\zeta_{1}$ and $\zeta_{2}$, do not directly appear in Equation (19), they influence $R_{\mathrm{d} 1}$ and $R_{\mathrm{d} 2}$, respectively. Therefore, it may be expected that the trends for variation of modal deformations would be directly related to how the plan-wise distribution of supplemental damping affects the apparent modal damping ratios.

\section{EFFECTS OF SYSTEM PARAMETERS ON MODAL DEFORMATIONS}

Translational and rotational components of the deformation at the $\mathrm{CM}$ due to each modal pair were computed using Equation (19) and are presented in Figure 9. Results were generated for two cases: $\beta_{1}=1$ and $\beta_{2}=1$. In the first case, $\beta_{2}$ would be smaller than one for which deformation response factor $R_{\mathrm{d} 2}$ would be close to one, i.e. response due to the second modal pair would be amplified very little due to dynamics of the system. On the other hand, response due to the first modal pair would be significantly amplified because $R_{\mathrm{d} 1}$ for $\beta_{1}=1$ is much larger than one. Therefore, contribution of the second modal pair is much smaller than that of the first modal pair and only responses due to the first modal pair, $u_{y 1}$ and $a u_{\theta 1}$, are plotted (Figure 9(a)). For similar reasons, responses due to the second modal pair, $u_{y 2}$ and $a u_{\theta 2}$, are plotted in the second case (Figure 9(b)).

For $\beta_{1}=1$ (Figure 9(a)), both $u_{y 1}$ and $a u_{\theta 1}$ are the smallest for $\bar{e}_{\mathrm{sd}}=-0.5$. They increase as the CSD moves from the left to right, i.e., $\bar{e}_{\text {sd }}$ varies from -0.5 to 0.5 and reach their maximum value 


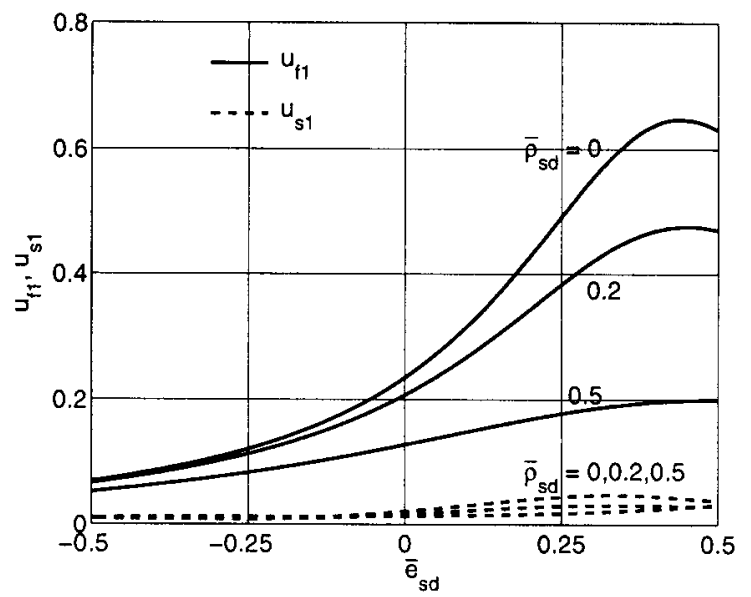

Figure 10. Deformations at the flexible and stiff edge of asymmetric-plan systems with supplemental damping due to first modal pair: $\beta_{1}=1$.

near $\bar{e}_{\mathrm{sd}}=0.5$. For $\beta_{2}=1$ (Figure 9(b)), $u_{y 2}$ and $a u_{\theta 2}$, are the smallest for $\bar{e}_{\mathrm{sd}}=0.5$ and tend to increase as the CSD moves from the right to left, i.e., $\bar{e}_{\text {sd }}$ varies from 0.5 to -0.5 . In this case, the maximum value occurs between $\bar{e}_{\mathrm{sd}}=0$ and -0.5 . These effects are the most pronounced for $\bar{\rho}_{\mathrm{sd}}=0$ and diminish as $\bar{\rho}_{\mathrm{sd}}$ becomes larger. The values of $u_{y 1}$ and $a u_{\theta 1}$ for the first case are much larger than values of $u_{y 2}$ and $a u_{\theta 2}$ in the second. This indicates that deformations are controlled by the first case, i.e., when the forcing frequency is close to the first modal frequency.

The above noted effects are directly related to the trends observed earlier for the deformation response factors, $R_{\mathrm{d} 1}$ and $R_{\mathrm{d} 2}$ (Figure 6). This is to be expected because deformation response factor is the only parameter that is significantly affected by the plan-wise distribution of damping; as noted previously, all other parameters (Equation (19)) are influenced very little.

The presented results indicate that if the first modal pair dominates the response, as would be the case for $\beta_{1}=1$, the smallest deformation response occurs when $\bar{e}_{\text {sd }}$ is as close to -0.5 as possible. If the second modal pair dominates the response, as would be the case for $\beta_{2}=1$, the smallest deformation response occurs when $\bar{e}_{\text {sd }}$ is as close to 0.5 as possible. Therefore, the plan-wise distribution of the supplemental damping to obtain the most reduction in the deformation response, i.e., selection of $\bar{e}_{\text {sd }}$, should depend on which of the two modal pairs dominates the response. If the first modal pair dominates, the supplemental damping should be distributed such that the CSD is as far away from the CM, on the side opposite to the CR, as possible, i.e., $\bar{e}_{\text {sd }}$ as close to -0.5 as possible. If the second modal pair dominates, then the supplemental damping should be distributed such that the CSD is as far away from the CM, on the same side of the CR, as possible, i.e., $\bar{e}_{\text {sd }}$ as close to 0.5 as possible.

Figure 10 presents the deformations at the flexible and stiff edges of the system, $u_{\mathrm{s} 1}$ and $u_{\mathrm{f} 1}$, due to the first modal pair for $\beta_{1}=1$. As expected, deformations on the flexible edge, $u_{\mathrm{f} 1}$, are much larger than those on the stiff edge, $u_{\mathrm{s} 1}$. The edge deformations are the smallest for $\bar{e}_{\mathrm{sd}}=-0.5$. They increase as the CSD moves from the left to right, i.e., $\bar{e}_{\mathrm{sd}}$ varies from -0.5 to 0.5 and reach their maximum value near $\bar{e}_{\text {sd }}=0.5$. These trends are similar to those noted earlier for $u_{y 1}$ and 
$a u_{\theta 1}$ (Figure 9(a)). Although results are not presented for reasons of brevity, the opposite trends may be expected for $\beta_{2}=1$.

The dependence of the edge deformations on $\bar{e}_{\mathrm{sd}}$ as well as $\bar{\rho}_{\mathrm{sd}}$ is the largest for $\bar{e}_{\mathrm{sd}}>0$. For $\bar{e}_{\text {sd }}<0$, especially for values of $\bar{e}_{\text {sd }}$ between -0.25 and -0.5 , the edge deformations are affected very little by either $\bar{e}_{\text {sd }}$, as indicated by flattening of the curves, or by $\bar{\rho}_{\text {sd }}$, as apparent from closeness of the three curves for $\bar{\rho}_{\text {sd }}=0,0.2$ and 0.5 .

Since one of the major concerns for asymmetric plan buildings is to reduce deformations on the flexible edge, the plan-wise distribution of the supplemental damping should be such that deformation of this edge are reduced the most. The presented results suggests that this objective would be met if the supplemental damping is distributed such the CSD is as far away from the CM, on the side opposite to the $\mathrm{CR}$, as possible, i.e. $\bar{e}_{\mathrm{sd}}$ as close to -0.5 as possible, and $\bar{\rho}_{\mathrm{sd}}$ is as large as possible. Such a distribution corresponds to maximizing the apparent modal damping in the first mode (Figure 2(b)). Since value of $\bar{e}_{\text {sd }}$ as close to -0.5 as possible and largest value of $\bar{\rho}_{\text {sd }}$ cannot be physically obtained simultaneously, it may be sufficient to distribute supplemental damping such that $\bar{e}_{\text {sd }}$ is equal in magnitude but opposite in algebraic sign to the structural eccentricity. This distribution leads to near optimal reduction in the flexible edge deformation; additional reductions, although possible, are small because of the low sensitivity of the deformation in this range of system parameters.

\section{CONCLUSIONS}

This paper presents the necessary theoretical background for computing the response of asymmetric-plan buildings with supplemental viscous damping to harmonic ground motion using modal analysis techniques. First, various modal properties - apparent modal periods, apparent damping ratios, mode shape components, modal participation factors and dynamic amplification factors - are computed and their variation with the plan-wise distribution of damping investigated leading to the following conclusions:

1. Most modal parameters, except dynamic amplification factor, are affected very little by the plan-wise distribution of supplemental damping. This is especially true for practical range of the system parameters.

2. Dynamic amplification factor (DAF) is significantly affected by the plan-wise distribution of supplemental damping. If the forcing frequency is close to the first apparent frequency, i.e., $\beta_{1}=1, R_{\mathrm{d} 1}$ increases as CSD moves from left to right of the system plan, i.e., as $\bar{e}_{\mathrm{sd}}$ varies from -0.5 to 0.5 . If the forcing frequency is close to the second apparent frequency, i.e., $\beta_{2}=1, R_{\mathrm{d} 2}$ increases as CSD moves from right to left of the system plan, i.e., as $\bar{e}_{\mathrm{sd}}$ varies from 0.5 to -0.5 , DAF becomes smaller with larger the value of $\bar{\rho}_{\mathrm{sd}}$.

3. The trends for DAF are directly related to the apparent modal damping ratios, $\zeta_{1}$ and $\zeta_{2}$. $\zeta_{1}$ increases and $\zeta_{2}$ decreases as CSD moves from right to left to the system plan, i.e., as $\bar{e}_{\text {sd }}$ varies from 0.5 to -0.5 . Both $\zeta_{1}$ and $\zeta_{2}$ become larger as $\bar{\rho}_{\text {sd }}$ increases.

Subsequently, effects of the plan-wise distribution of supplemental damping on steady-state modal deformations - translational and rotational components of deformations at the CM, and flexible- and stiff-edge deformations - are examined. It is shown that

1. The smallest deformation response at the $\mathrm{CM}$ and at the edges occurs when (a) $\bar{e}_{\mathrm{sd}}$ is as close to -0.5 as possible for $\beta_{1}=1$ and (b) $\bar{e}_{\mathrm{sd}}$ is as close to 0.5 as possible for $\beta_{2}=1$. 
2. For obtaining the largest reduction in the flexible edge deformation, which is generally the most critical edge, the supplemental damping should be distributed such the CSD is as far away from the CM, on the side opposite to the CR, as possible and $\bar{\rho}_{\text {sd }}$ is as large as possible. Since both these criteria cannot be physically satisfied simultaneously, it may be sufficient to distribute supplemental damping such that $\bar{e}_{\text {sd }}$ is equal to in magnitude but opposite in algebraic sign to the structural eccentricity.

3. The trends for modal deformations are directly related to the apparent modal damping ratios.

The key understanding that has been developed in this investigation is the influence of the plan-wise distribution of the supplemental damping on the apparent modal damping ratio. Since the trends for modal deformations are directly related to the apparent modal damping ratios, it is likely that simplified procedures suitable for use in the design practice would evolve from simplified calculation of these damping ratios. Research in this direction is in progress and would be reported later.

\section{APPENDIX}

Steady-state response of a non-classically damped system in the $n$th complex modal pair is

$$
\hat{z}_{k n}(t)=\left(\Gamma_{n} \bar{H}_{n} \Phi_{k n}+\Gamma_{n}^{*} \tilde{H}_{n} \Phi_{k n}^{*}\right) \ddot{u}_{\mathrm{go}} \mathrm{e}^{\mathrm{j} \omega t}
$$

in which $\Gamma_{n}$ and $\Gamma_{n}^{*}$ are the complex-conjugate pair of modal participation factor; $\Phi_{k n}$ and $\Phi_{k n}^{*}$ are the complex-conjugate pair of the mode shape component at the $k$ th degree of freedom; and $\bar{H}_{n}$ and $\widetilde{H}_{n}$ are the complex frequency response functions given as

$$
\bar{H}_{n}=\frac{1}{\lambda_{n}+\mathrm{j} \omega} \quad \text { and } \quad \tilde{H}_{n}=\frac{1}{\lambda_{n}^{*}+\mathrm{j} \omega}
$$

where $\omega$ is the frequency of the harmonic ground motion; and $\lambda_{n}$ and $\lambda_{n}^{*}$ are the complex conjugate pair of eigenvalues given by Equation (8).

Recognizing that a complex number $x=a+\mathrm{j} b$ and its complex-conjugate $x^{*}=a-\mathrm{j} b$ can be expressed in the exponential form as

$$
x=|x| \mathrm{e}^{\mathrm{j} \theta} \quad \text { and } \quad x^{*}=|x| \mathrm{e}^{-\mathrm{j} \theta}
$$

in which $|x|=\sqrt{a^{2}+b^{2}}$ represents magnitude of the complex number and $\theta=\tan ^{-1}(b / a)$ represents the associated phase angle, $z_{k n}(t)$ can be expressed as

$$
\hat{z}^{k n}(t)=\left|\Gamma_{n}\right| \times\left|\Phi_{k n}\right| \times\left(\bar{H}_{n} \mathrm{e}^{\mathrm{j}\left(\theta_{n}^{\Gamma}+\theta_{k n}^{\Phi}\right)}+\tilde{H}_{n} \mathrm{e}^{-\mathrm{j}\left(\theta_{n}^{\Gamma}+\theta_{k n}^{\Phi}\right)}\right) \ddot{u}_{\mathrm{go}} \mathrm{e}^{\mathrm{j} \omega t}
$$

in which $\left|\Gamma_{n}\right|$ and $\left|\Phi_{k n}\right|$ are magnitudes (or absolute values) of the modal participation factor and the mode shape component, respectively; and $\theta_{n}^{\Gamma}$ and $\theta_{n}^{\Phi}$ are the associated phase angles. Utilizing the definition of complex-frequency response functions (Equation (A2)), the bracketed term in 
Equation (A4) can be written as

$$
\left(\bar{H}_{n} \mathrm{e}^{\mathrm{j}\left(\theta_{n}^{\Gamma}+\theta_{k n}^{\Phi}\right)}+\tilde{H}_{n} \mathrm{e}^{-\mathrm{j}\left(\theta_{n}^{\Gamma}+\theta_{k n}^{\Phi}\right)}\right)=\frac{1}{\left(\lambda_{n}+\mathrm{j} \omega\right)\left(\lambda_{n}^{*}+\mathrm{j} \omega\right)}\left[\left(\lambda_{n}^{*}+\mathrm{j} \omega\right) \mathrm{e}^{\mathrm{j}\left(\theta_{n}^{\Gamma}+\theta_{k n}^{\Phi}\right)}+\left(\lambda_{n}+\mathrm{j} \omega\right) \mathrm{e}^{-\mathrm{j}\left(\theta_{n}^{\Gamma}+\theta_{k n}^{\Phi}\right)}\right]
$$

Using the eigenvalue expressions of Equation (8), the term outside the square brackets in Equation (A5) can be expressed as

$$
\frac{1}{\left(\lambda_{n}+\mathrm{j} \omega\right)\left(\lambda_{n}^{*}+\mathrm{j} \omega\right)}=\frac{1}{\omega_{n}^{2}} R_{\mathrm{dn}} \mathrm{e}^{\mathrm{j} \theta_{n}^{R}}
$$

with

$$
R_{\mathrm{d} n}=\frac{1}{\sqrt{\left(1-\beta_{n}^{2}\right)^{2}+\left(2 \zeta_{n} \beta_{n}\right)^{2}}} \quad \text { and } \quad \theta_{n}^{R}=\tan ^{-1}\left(\frac{2 \zeta_{n} R_{n}}{1-\beta_{n}^{2}}\right)
$$

where $\beta_{n}=\omega \div \omega_{n}$. The term inside the square brackets of Equation (A5) can be separated into two parts as

$\left(\lambda_{n}^{*}+\mathrm{j} \omega\right) \mathrm{e}^{\mathrm{j}\left(\theta_{n}^{\Gamma}+\theta_{k n}^{\Phi}\right)}+\left(\lambda_{n}+\mathrm{j} \omega\right) \mathrm{e}^{-\mathrm{j}\left(\theta_{n}^{\Gamma}+\theta_{k n}^{\Phi}\right)}=\lambda_{n}^{*} \mathrm{e}^{\mathrm{j}\left(\theta_{n}^{\Gamma}+\theta_{k n}^{\Phi}\right)}+\lambda_{n} \mathrm{e}^{-\mathrm{j}\left(\theta_{n}^{\Gamma}+\theta_{k n}^{\Phi}\right)}+\mathrm{j} \omega\left(\mathrm{e}^{\mathrm{j}\left(\theta_{n}^{\Gamma}+\theta_{k n}^{\Phi}\right)}+\mathrm{e}^{-\mathrm{j}\left(\theta_{n}^{\Gamma}+\theta_{k n}^{\Phi}\right)}\right)$

Utilizing exponential forms of $\lambda_{n}=\omega_{n} \mathrm{e}^{\mathrm{j} \theta_{n}^{\lambda}}$ with $\theta_{n}^{\lambda}=\tan ^{-1}\left(-\sqrt{1-\zeta_{n}^{2}} /-\zeta_{n}\right)$, the first term in Equation (A8) simplifies to

$$
\lambda_{n}^{*} \mathrm{e}^{\mathrm{j}\left(\theta_{n}^{\Gamma}+\theta_{k n}^{\Phi}\right)}+\lambda_{n} \mathrm{e}^{-\mathrm{j}\left(\theta_{n}^{\Gamma}+\theta_{k n}^{\Phi}\right)}=\omega_{n}\left(\mathrm{e}^{\mathrm{j}\left(\theta_{n}^{\Gamma}+\theta_{k n}^{\Phi}-\theta_{n}^{\lambda}\right)+} \mathrm{e}^{-\mathrm{j}\left(\theta_{n}^{\Gamma}+\theta_{k n}^{\Phi}-\theta_{n}^{\lambda}\right)}\right)
$$

By using de Moivre's theorem and the simplification achieved in Equation (A9), (A8) can be expressed as

$\left(\lambda_{n}^{*}+\mathrm{j} \omega\right) \mathrm{e}^{\mathrm{j}\left(\theta_{n}^{\Gamma}+\theta_{k n}^{\Phi}\right)}+\left(\lambda_{n}^{*}+\mathrm{j} \omega\right) \mathrm{e}^{-\mathrm{j}\left(\theta_{n}^{\Gamma}+\theta_{k n}^{\Phi}\right)}=2 \omega_{n}\left[\cos \left(\theta_{n}^{\Gamma}+\theta_{k n}^{\Phi}-\theta_{n}^{\lambda}\right)+\mathrm{j} \beta_{n} \cos \left(\theta_{n}^{\Gamma}+\theta_{k n}^{\Phi}\right)\right]$

Utilizing the exponential form, the term inside the square brackets of Equation (A10) can be rewritten as

$$
\cos \left(\theta_{n}^{\Gamma}+\theta_{k n}^{\Phi}-\theta_{n}^{\lambda}\right)+\mathrm{j} \beta_{n} \cos \left(\theta_{n}^{\Gamma}+\theta_{k n}^{\Phi}\right)=C_{k n} \mathrm{e}^{\mathrm{j} \theta_{k n}^{c}}
$$

with

$$
\begin{aligned}
C_{k n} & =\sqrt{\left(\cos \left(\theta_{n}^{\Gamma}+\theta_{k n}^{\Phi}-\theta_{n}^{\lambda}\right)\right)^{2}+\left(\beta_{n} \cos \left(\theta_{n}^{\Gamma}+\theta_{k n}^{\Phi}\right)\right)^{2}} \\
\theta_{k n}^{C} & =\tan ^{-1}\left(\frac{\beta_{n} \cos \left(\theta_{n}^{\Gamma}+\theta_{k n}^{\Phi}\right)}{\cos \left(\theta_{n}^{\Gamma}+\theta_{k n}^{\Phi}-\theta_{n}^{\lambda}\right)}\right)
\end{aligned}
$$

Combining all the results obtained so far leads to Equation (19). 


\section{ACKNOWLEDGEMENTS}

This research investigation is funded by the National Science Foundation under Grant CMS-9812414. This financial support is gratefully acknowledged.

\section{REFERENCES}

1. Hejal R, Chopra AK. Earthquake response of torsionally-coupled buildings. Report UCB/EERC-·87/20, Earthquake Engineering Research Center, University of California, Berkeley, CA, 1987.

2. Goel RK, Chopra AK. Inelastic seismic response of one-storey, asymmetric-plan systems. Report UCB/EERC-90/14, Earthquake Engineering Research Center, University of California, Berkeley, CA, 1990.

3. Rutenberg A. Nonlinear response of asymmetric building structures and seismic codes: a state of the art review. European Earthquake Engineering, 1992; VI(2):3-19.

4. International Association for Earthquake Engineering. Earthquake Resistant Regulations, A World List, 1992, Tokyo, 1992.

5. Aiken ID, Kelly JM. Earthquake simulator testing and analytical studies of two energy-absorbing systems for multistorey structures. Report UCB/EERC-90/03, Earthquake Engineering Research Center, University of California, Berkeley, CA, 1990.

6. Chang KC. Seismic behavior of steel frame with added viscoelastic dampers. Journal of Structural Engineering 1995; 121(10): $1418-1426$.

7. Constantinou MC, Symans MD. Experimental and analytical investigation of seismic response of structures with supplemental fluid viscous dampers. Report No. NCEER-92-0032, National Centre for Earthquake Engineering Research, Buffalo, NY, (1992).

8. Gluck N, Reinhorn AM, Gluck J, Levy R. Design of supplemental dampers for control of structures. Journal of Structural Engineering, ASCE, 1996, 122(12):1394-1399.

9. Hanson RD. Supplemental damping for improved seismic performance. Earthquake Spectra, 1993, 9(3):319-334.

10. Kasai K, Munsi JA. Seismic response of viscoelastic frame with yielding members. Proceedings of 5th National Conference on Earthquake Engineering, vol. I, Earthquake Engineering Research Institute, Oakland, CA, 1994; 839-848.

11. Pall AS. Energy dissipation devices for aseismic design of buildings. Proceedings of a Seminar and Workshop on Base Isolation and Passive Energy Dissipation, ATC-17, Applied Technology Council, Palo Alto, California, 1986; 223-232.

12. Tsopelas P, Okamoto S, Constantinou MC, Ozaki D, Fuji S. Experimental and analytical study of systems consisting of sliding bearing, rubber restoring force devices, and fluid dampers. Report No. NCEER-94-0002, National Center for Earthquake Engineering Research, Buffalo, NY, 1994.

13. Rasmussen E. Dampers hold sway. Civil Engineering, 1997; 67(3):40-43.

14. Arista A, Gomez R. Influence of energy dissipation devices on the torsional response of single-storey structures. Proceedings of a Seminar and Workshop on Base Isolation and Passive Energy Dissipation, ATC-17-1, Applied Technology Council, vol. 2, 1993; 651-662.

15. Martin L, Pekau OA. Improved performance of friction damped asymmetric structures. Proceedings of the 5th Canadian Conference on Earthquake Engineering, Montreal, 1995; 927-934.

16. Pekau OA, Guimond R. Controlling seismic response of eccentric structures by friction dampers. Earthquake Engineering and Structural Dynamics, 1991; 20:505-521.

17. Li ZX, He YA. Optimal damper control for 3-dimensional tall buildings under earthquake. Proceedings of the 10th World Conference on Earthquake Engineering, vol. 7, 1992; 4159-4164.

18. Goel RK. Effects of supplemental viscous damping on seismic response of asymmetric-plan systems. Earthquake Engineering and Structural Dynamics, 1997; 27:125-141.

19. Goel RK. Control of earthquake-induced torsional vibrations in asymmetric buildings. Proceedings of the 2nd World Conference on Structural Control, Kyoto, Japan, vol. 2, 1998; 1623-1630.

20. Goel RK. Effects of supplemental damping on earthquake response of asymmetric buildings. CD-ROM Proceedings of the 6th U.S. National Conference on Earthquake Engineering, Seattle, 1998.

21. Goel RK. Seismic control of asymmetric structures. Proceedings of the 1999 Structures Congress, New Orleans, 1999; $15-18$.

22. Inman DJ. Engineering Vibrations. Prentice-Hall: Englewood Cliffs, NJ, 1996.

23. Humar JL. Dynamics of Structures. Prentice-Hall: Englewood Cliffs, NJ, 1990.

24. Chopra AK. Dynamics of Structures: Theory and Applications to Earthquake Engineering. Prentice-Hall, Upper Saddle River, NJ, 1995.

25. Clough RW, Penzien JP. Dynamics of Structures (2nd edn). McGraw-Hill: New York, 1993.

26 Kreyszig E. Advanced Engineering Mathematics (7th edn), Wiley: New York, 1993. 